\title{
VARIABILIDADE NAS TAXAS FISIOLÓGICAS DO MEXILHÃO PERNA PERNA EM DOIS SÍTIOS DE CULTIVO DO LITORAL NORTE DE SANTA CATARINA.
}

\author{
RESGALLA Jr., C.; MANZONI, G.; KUROSHIMA, K.N.; REIS Fo., R.W.* \& K.S. LAITANO* \\ Centro de Ciências Tecnológicas da Terra e do Mar (CTTMar) \\ Universidade do Vale do Itajaí (UNIVALI) \\ R. Uruguai, 458 - Cx.P. 360 - Itajaí, SC - 88.302-202 \\ * Bolsistas PIBIC (CNPq/UNIVALI)
}

\begin{abstract}
RESUMO
Com a crescente atividade sócio-econômica da mitilicultura na costa do estado de Santa Catarina, problemas relacionados com a identificação de novos sítios de cultivo tornaram-se prioritários. Dentre os mais diferentes métodos utilizados para análise de potenciais regiões produtoras, o estudo das taxas fisiológicas dos mexilhões enquadram-se como uma das mais adequadas, incluindo neste caso, o monitoramento de sítios já estabelecidos e problemas relacionados com a poluição ambiental. Este relatório faz referência a uma preliminar comparação entre duas áreas de cultivo do mexilhão Perna perna no litoral norte do estado (municípios de Penha e Bombinhas). Dados ambientais de temperatura, salinidade, material em suspensão e fisiológicos do mexilhão como crescimento, filtração, respiração e assimilação foram comparados. Os principais resultados deste estudo podem ser resumidos em: relação direta entre a temperatura e a taxa de crescimento e relação entre a salinidade e a taxa de respiração para os dois ambientes. O material em suspensão apresentou uma maior variabilidade ambiental influenciada pelos aportes continentais mas que necessitam de maiores investigações. A importância das taxas de maturação dos folículos embrionários dos mexilhões foi sugerida na relação com as taxas de filtração e assimilação. Em termos de produção, os ambientes mostraram maiores diferenças nos meses frios e similar resultados nos meses quentes. Entretanto, resultados conclusivos estão dependentes de estudos ao longo de um ciclo anual.
\end{abstract}

Palavras Chave: Perna perna, Taxa de Crescimento, Taxa de Filtração, Taxa de Respiração, Taxa de Assimilação.

\section{PHYSIOLOGICAL VARIABILITY OF THE MUSSEL PERNA PERNA IN TWO CULTIVATION SITES IN THE NORTH COAST OF SANTA CATARINA STATE.}

\begin{abstract}
With the growing socioeconomic activity of mussel farming on Santa Catarina's coast, the identification of new cultivation sites became necessary. Among a variety of methods used for the analysis of potential producing areas, studying of the mussel physiological rates may be considered as one of the most appropriated, specially when addressing the monitoring of sites already established and the negative effects of environmental pollution. This report presents a preliminary comparison between two areas of cultivation in the northern coast of the state (municipalities of Penha and Bombinhas). In both locations, environmental data (temperature, salinity and seston) and physiological parameters of the mussels (growth, and filtration, respiration and assimilation rates) were analysed. Our findings suggested a direct relationship between temperature and mussel growth rate and also between salinity and respiration rates for both areas. The seston concentration showed a high environmental variability as a response to the continental drainage. In addition, the filtration and
\end{abstract}


assimilation rates seemed to be influenced by the stages of embryonic follicle maturation. In terms of production, the sites showed larger differences during the colder months as compared to the warmer ones, when the results were quite similar. More conclusive results are dependent of studies conducted along a complete annual cycle.

Key Words: Perna perna, Growth Rate, Filtration Rate, Respiration Rate, Assimilation Rate.

\section{INTRODUÇÃO}

O conhecimento dos taxas fisiológicas de moluscos, como os mexilhões Perna perna, que apresentam uma potencial aplicação como bioindicadores é de fundamental importância para o desenvolvimento de programas de monitoramento "in situ" direcionados tanto para estudos de impacto ambiental (EPA, 1989), como em análises da produção de futuras áreas destinadas a implantação da mitilicultura.

Os estudos destes parâmetros fisiológicos em bivalves, a nível mundial, estão relativamente bem investigados e explorados, diferenciando-se entretanto quanto à espécie investigada. Destacam-se o marisco do hemisfério norte Mytilus edulis, Cloromytilus clorus, Mercenaria mercenaria, entre outros.

Os estudos fisiológicos em bivalves normalmente concentram-se nas taxas de filtração. Testes a diferentes temperaturas (Villiers et al., 1989 e Kryger \& Riisgard, 1988), salinidades (Navarro, 1988), velocidade de fluxo da água (Wildish \& Miyares, 1990), concentração (Winter, 1973) do alimento e seus efeitos sobre a taxa de assimilação são bem conhecidos (Werner \& Hollibaugh, 1993). Cabe destacar os extensivos trabalhos de Winter (1978), Newell (1979) e Bayne \& Newell (1983) sobre as taxas de filtração, assimilação, respiração e suas variações com relação a diferentes concentrações de alimento, diâmetro do alimento oferecido, tipo de alimento (seleção), temperatura, aclimatação, tamanho dos bivalves, assim como observações na eficiência de retenção e formação de pseudo-fezes destinados a investigações em sistemas artificiais em aquicultura.
Trabalhos cuja integração dos parâmetros fisiológicos para os cálculos do potencial de crescimento são, entretanto, relativamente limitados (Grant \& Cranford, 1991 e Widdows \& Johnson, 1988) e pouco tem contribuído nos estudos efetivos na avaliação da qualidade ambiental.

O presente relatório comparou as taxas de filtração, assimilação e respiração do mexilhão Perna perna entre dois sítios de cultivo do litoral norte de Santa Catarina a fim de avaliar a metodologia empregada e possíveis diferenças da produção entre as áreas selecionadas.

\section{MATERIAL E MÉTODOS}

Um total de 150 exemplares do mexiIhão Perna perna (tamanho aproximado de 30 $\mathrm{mm}$ ) foram obtidos das cordas dos cultivos suspensos da Enseada de Armação do Itapocory, município de Penha, litoral norte de Santa Catarina. Duas cordas de cultivo com 75 exemplares de mexilhão em cada corda, previamente medidos, foram montadas e catalogadas para aclimatação no próprio local de origem (Penha) e outra destinada a área de cultivo do município de Bombinhas (20 mn ao sul de Penha) por 30 dias. Esta técnica foi realizada nos meses de junho, setembro, outubro e dezembro de 1997 e fevereiro de 1998.

Após o período de aclimatação, os organismos foram transportados ao Laboratório de Cultivo de Organismos Marinhos (CTTMar/UNIVALI) e mantidos à temperatura constante $\left(20 \pm 2^{\circ} \mathrm{C}\right)$, em inanição (starvation) por um período de 24 horas antes dos testes. Todos os experimentos foram realizados a temperatura de laboratório, salinidade de 30 a $35 \%$, no escuro, em 10 
réplicas para cada ambiente e parâmetro fisiológico, em frascos plásticos de 1,0 litro de volume. Os testes de filtração apresentaram uma duração de 1 hora enquanto que a respiração e assimilação, 3 horas.

\section{Taxa de Filtração (TF)}

Utilizou-se a técnica desenvolvida para determinação da taxa de filtração de organismos suspensívoros-filtradores zooplanctônicos (Omori \& Ikeda, 1984). Os testes foram conduzidos em água do mar filtrada em filtros de fibra de vidro de $0,5 \mathrm{~mm}$ de porosidade e com inoculação de uma concentração conhecida de fitoplâncton (Chaetoceros gracilis). Frascos controle foram mantidos nas mesmas condições, porém sem mexilhões. No início e final do teste foram retirada alíquotas de $50 \mathrm{ml}$ dos frascos teste, e imediatamente feito a leitura da absorbância em espectrofotômetro (Shimadzu UV-160A) a $750 \mathrm{~nm}$. Em cada câmara teste foi introduzido um organismo teste. As taxas de filtração foram estimadas utilizando-se a seguinte equação:

$$
T F=\frac{v}{n} \times\left[\left(\frac{\ln C_{0}-\ln C_{t}}{t}\right)-f\right]
$$

onde:

TF = Taxa de Filtração (litros.hora-1).

$\mathrm{n}=$ número de organismos por frasco teste.

$\mathrm{v}=$ volume do frasco teste (litros).

In $\mathrm{C}_{0}=$ Logaritmo neperiano da absorbância do fitoplâncton inoculado.

In $\mathrm{C}_{\mathrm{t}}=$ Logaritmo neperiano da absorbância do fitoplâncton no tempo t.

$\mathrm{t}=$ tempo de incubação (hora).

$f=$ fator de correção, calculado pela mesma fórmula para os frascos controle, e relacionado com a decantação do fitoplâncton.

Os valores das TF foram expressos em I. $\mathrm{h}^{-1} \cdot \mathrm{g}^{-1}$ do peso seco dos organismos incubados.

\section{Taxa de Respiração (TR)}

Os testes foram conduzidos nas mesmas condições apresentadas para a TF, sendo os mexilhões mantidos com fitoplâncton (alimento) durante 3 horas, em água saturada de oxigênio. $O$ declínio do oxigênio dissolvido foi medido mediante o uso de um oxímetro digital (YSI mod. 58). Frascos controle (sem mexilhões) foram monitorados para correções dos dados obtidos.

Os cálculos seguiram a equação proposta por Widdows \& Johnson (1988):

$$
T R=\left\{\left[C_{(t 0)}-C_{(t i)}\right] \times \frac{v}{\Delta t}\right\}-f
$$

onde:

$\mathrm{TR}=$ Taxa de Respiração $\left(\mathrm{ml} \mathrm{O}_{2} \cdot\right.$ hora $\left.^{-1}\right)$.

$\mathrm{C}_{(\mathrm{t} 0)}=$ concentração do oxigênio no tempo zero $\left(\mathrm{ml} \mathrm{O}_{2} \cdot \mathrm{l}^{-1}\right)$.

$\mathrm{C}_{\text {(ti) }}=$ concentração do oxigênio no tempo i $\left(\mathrm{ml} \mathrm{O}_{2} \cdot \mathrm{I}^{-1}\right)$.

$\mathrm{v}=$ volume do frasco teste (litros).

$\mathrm{Dt}$ = intervalo de tempo de incubação.

$f=$ fator de correção nos frascos controle.

As taxas foram expressas em $\mathrm{mg}$ de Oxigênio. $\mathrm{h}^{-1} \cdot \mathrm{g}^{-1}$ do peso seco dos organismos.

\section{Taxa de Assimilação (TA)}

Os testes foram realizados nas mesmas condições da TF e da TR. O cálculo, proposto por Omori \& Ikeda (1984), consistiu em comparar o percentual do conteúdo de matéria orgânica presente no fitoplâncton oferecido com o percentual da matéria orgânica presente nas fezes coletadas no fundo do frasco teste após 3 horas segundo a equação:

$$
T A=\left[\frac{(I-F)}{(1-F) \times I}\right] \times 100 \%
$$


onde:

TA = Taxa de Assimilação (\%)

I = razão entre o peso de matéria orgânica sobre o peso seco do fitoplâncton oferecido (\%).

$\mathrm{F}=$ razão entre o peso de matéria orgânica sobre o peso seco das fezes produzidas (\%).

Os conteúdos de matéria orgânica, tanto no fitoplâncton como nas fezes, foram estimados por combustão em forno mufla a $500^{\circ} \mathrm{C}$ por duas horas (Beers, 1976), técnica conhecida como "peso seco livre de cinzas".

\section{Biomassa dos Organismos Teste}

Após os testes fisiológicos, os organismos foram medidos e separados os tecidos das conchas. O peso seco dos tecidos internos foram estimados por secagem em estufa a $60^{\circ} \mathrm{C}$ por 24 horas (Beers, 1976).

\section{Taxa de Crescimento (TC)}

Estimativas da taxa de crescimento instantânea (g) foram realizadas mediante a variação do peso dos mexilhões no tempo inicial e final em cada ambiente em função do tempo de exposição. A equação utilizada foi:

$$
g=\frac{\left[\ln (\text { peso })-\ln \left(\text { peso }_{t-1}\right)\right]}{\Delta t}
$$

onde:

In $($ peso $)$ ) = logaritmo natural do peso seco do mexilhão no tempo $t$

In $\left(\right.$ peso $\left._{t-1}\right)=$ logaritmo natural do peso seco do mexilhão no tempo anterior

$\mathrm{D} t=$ intervalo de tempo

\section{Parâmetros Ambientais}

Dados de temperatura e salinidade dos dois sítios de cultivo foram obtidas com um multianalisador da marca Horiba (modelo U10). Dados do material em suspensão foram obtidos pela filtragem da água em filtros de fibra de vidro (GFF) e com secagem em estufa a $60^{\circ} \mathrm{C}$ por 12 horas (Strickland, \& Parsons, 1972).

\section{Análise Multivariada}

Análise de componentes principais (PCA) foram utilizadas nos dados ambientais e nas taxas fisiológicas, buscando relações entre as variáveis. O tratamento matemático seguiu as recomendações de Pielou (1984).

\section{RESULTADOS E DISCUSSÃO}

\section{Parâmetros ambientais}

As condições oceanográficas entre Penha e Zimbros foram similares, principalmente em termos de temperatura e salinidade (fig. 1). Entretanto, deve-se destacar que estes valores devem ser comparados com ressalvas, já que em Penha os estudos são muito mais intensos onde a obtenção de dados apresenta uma série temporal maior do que em Zimbros (CTTMar-UNIVALI - Projeto de Monitoramento Voluntário - Olho Vivo).

Em termos de material em suspensão, que poderia ser traduzido em biomassa disponível de alimento para os mexilhões, apresentou uma maior variabilidade. Como média geral, Penha apresentou MS superiores a Zimbros, com exceção do mês de setembro.

Estes resultados podem ser explicados pela proximidade entre os dois sítios de cultivo para a temperatura e salinidade mas sob diferentes aportes continentais. Em Zimbros o maior aporte seria oriundo do Rio Tijucas $\left(58 \mathrm{~m}^{3} \cdot \mathrm{s}^{-1}\right)$ enquanto que em Penha a influência seria do Rio Itajaí-Açu $\left(270 \mathrm{~m}^{3} . \mathrm{s}^{-1}\right)$, cuja bacia hidrográfica é a maior do estado.

\section{Taxas Fisiológicas}

Com exceção da taxa de respiração, os parâmetros fisiológicos do mexilhão entre 
as duas localidades foram similares (fig. 2). A taxa de crescimento foi oposta entre Zimbros e Penha nos meses de junho e setembro. No mês de junho a baixa taxa de crescimento em Penha foi concordante com a alta taxa de respiração, mostrando maior gasto energético no metabolismo basal o que provavelmente tenha desviado maior energia destinada para o crescimento (Bayne \& Newell, 1983). Em setembro, entretanto, a alta taxa de crescimento observada em Penha foi acompanhada somente pela diminuição na taxa de respiração.

\section{Análise Multivariada (Ambiente X Fisiolo- gia)}

Os resultados apresentados na figura 3 sugerem dois pontos principais: relação entre a temperatura, TC, TF e TA (primeiro eixo) e no segundo eixo relação entre a salinidade e a TR.

No segundo eixo, os mexilhões de Penha e Zimbros apresentaram relação entre salinidade e a taxa de respiração, mas de forma direta em Penha e inversa em Zimbros. Ou seja, quanto maior a salinidade em $\mathrm{Pe}$ nha maior é a respiração e quanto maior a salinidade em Zimbros menor é a respiração.
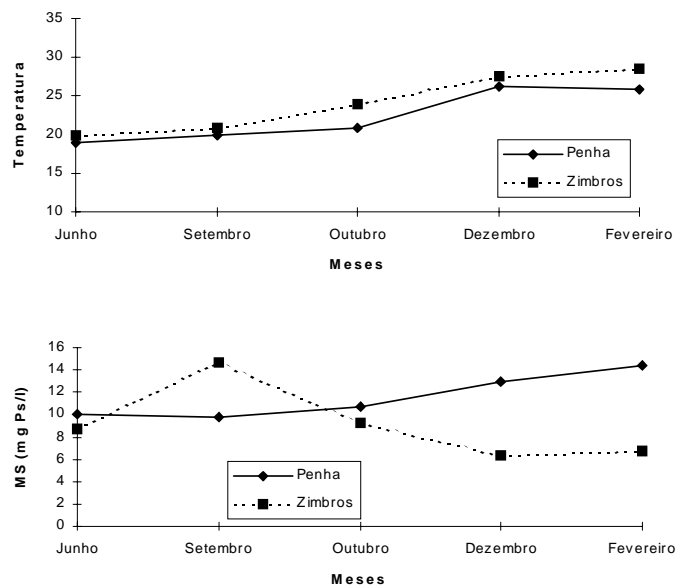

Independente dos dados de salinidade entre as duas localidades, esta análise sugere que os mexilhões de Penha estejam aclimatados a baixas salinidades, provavelmente sob influência do Rio Itajaí-Açu, e qualquer incremento resulta em um maior gasto metabólico na sua fisiologia interna. Por outro lado, em Zimbros, por ser uma área de cultivo sem uma severa influência de aportes continentais, os mexilhões estejam adaptadas a altas salinidades, o que resulta em um metabolismo basal menor em altas salinidades.

No primeiro eixo de variação, o fator ambiental claramente responsável pelo crescimento dos mexilhões foi a temperatura, fato já bastante estudado e conhecido em organismos pecilotérmicos (Bayne \& Newell, 1983 e Newell, 1979). Já o material em suspensão apresentou um comportamento diferenciado entre os ambient es. Em Penha apresentou relação direta com a TC e inversa em Zimbros. Estes resultados, somado a relação inversa entre a TC e as TF e TA, colocam em dúvida a aplicação do método. A TC é um indicativo da aplicação de parâmetros fisiológicos como taxas a serem usadas no potencial de crescimento (Scope for Growth) (EPA, 1989), e teoricamente uma relação inversa entre a TC

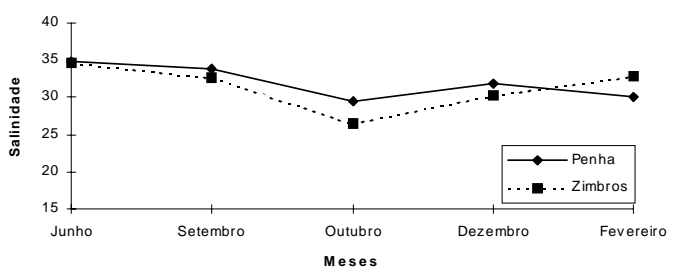

Figura 1 - Valores médios de temperatura $\left({ }^{\circ} \mathrm{C}\right)$, salinidade (\%) e material em suspensão (MS mg. $\mathrm{L}^{-1}$ ) de Penha e Zimbros para os meses analisados. 
com a TF e TA parece ser mais ou menos incoerente.

Estes resultados podem ser explicados, a princípio, por dois fatores:

1. Intensa infeção de parasitas nos mexilhões, que podem resultar em gastos adicionais da energia no metabolismo interno dos mexilhões (Fernandes, 1981). Este fato já está sendo investigado nas gônadas dos mexilhões (Bihl, em preparo) além da ocorrência de organismos planctônicos cujo ciclo de vida envolve estágios semi-parasitas em moluscos já registrados em Penha (Duarte \& Resgalla Jr., 1996).

2. A mais provável explicação entretanto seria um maior direcionamento da energia obtida pelo metabolismo para a maturação das gônadas (testículos e ovários), limitando assim as taxas de crescimento observadas. Esta
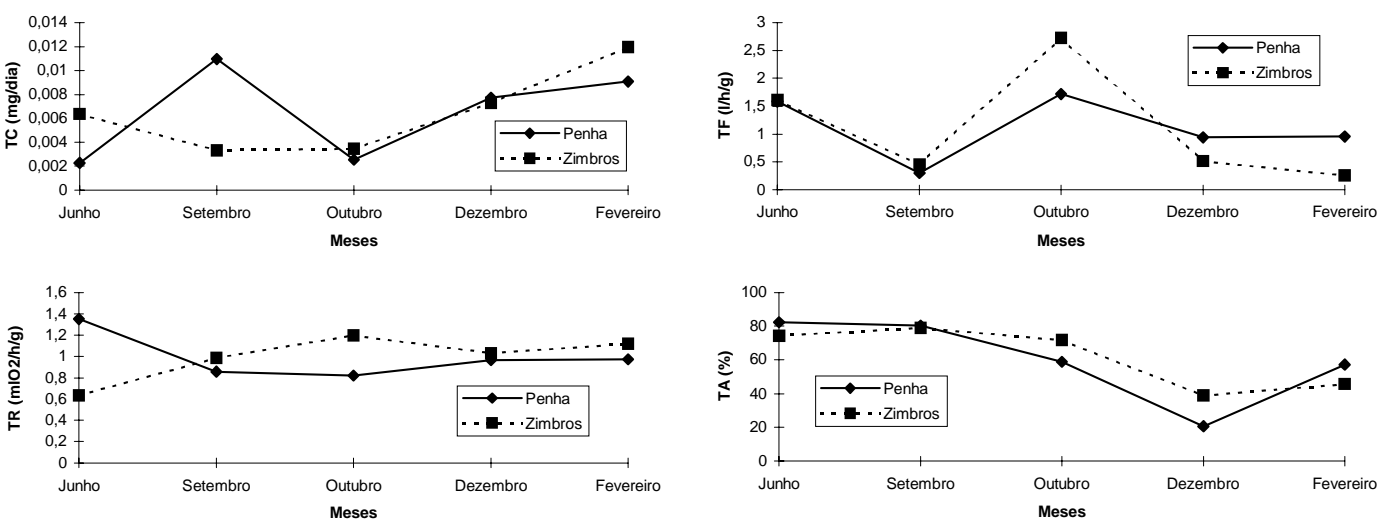

Figura 2 - Valores médios da taxa de crescimento ( $T C$ mg de peso seco.dia-1), taxa de filtração (TF litros.hora ${ }^{-1}$. grama $^{-1}$ ), taxa de respiração (TR mg de carbono.hora ${ }^{-1}$.grama ${ }^{-1}$ ) e taxa de assimilação (TA \%) de Penha e Zimbros para os meses analisados.

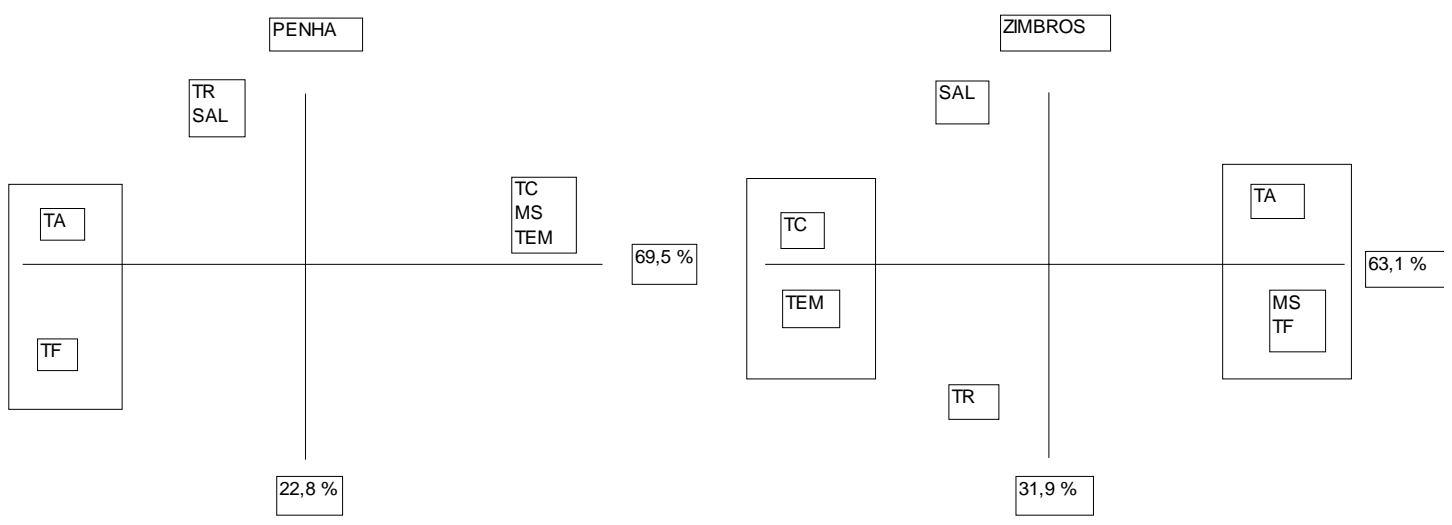

Figura 3 - Representação gráfica das análises de PCA para Penha e Zimbros, onde: TEM = temperatura, SAL = salinidade, $\mathrm{MS}=$ material em suspensão, $\mathrm{TC}=$ taxa de crescimento, $\mathrm{TF}=$ taxa de filtração, $\mathrm{TR}=$ taxa de respiração e TA $=$ taxa de assimilação. Os números referem-se a porcentagem da variância em cada eixo. 

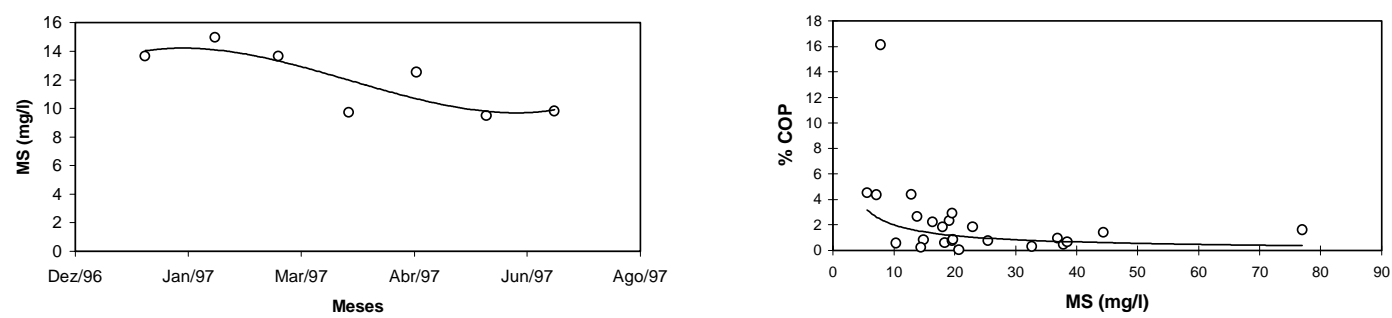

Figura 4 - Valores médios do material em suspensão e modelo matemático ao longo dos meses do ano de 1996/1997 (gráfico da esquerda) e relação entre material em suspensão (MS) e porcentagem de carbono orgânico particulado (COP) para o ano de 1998 sob influência de altas taxas de descarga do Rio Itajaí-Açu (gráfico da direita). Ambos os gráficos referem-se ao material em suspensão de Penha.

hipótese é ressaltada pelo tamanho dos mexilhões testados após sua aclimatação em campo, onde organismos com tamanho acima de $40 \mathrm{~mm}$ já estão em idade de reprodução (Wojciechowski Jr., 1997).

Um fator limitante para os cálculos do potencial de crescimento (Scope for Growth) neste estudo foi a falta de dados sobre o conteúdo orgânico do material em suspensão de ambas as localidades. Entretanto, esta análise passou a ser realizada rotineiramente pelo Laboratório de Oceanografia Química (CTTMar/UNIVALI) a partir do ano de 1998. Em Penha, onde os estudos são realizados mais intensamente, o material em suspensão parece mostrar uma sazonalidade ao longo do ano, enquanto que altos valores deste

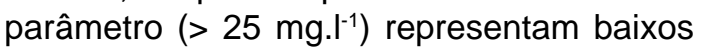
percentuais em conteúdo orgânico (figura 4). Esta variabilidade seguramente irá influir na produção dos sítios investigados assim como nas taxas fisiológicos estimadas.

\section{CONCLUSÕES E RECOMENDAÇÕES}

1. Os resultados apresentados sugerem uma produção (em termos de taxas de crescimento) similar entre os sítios de cultivos de Penha e Zimbros para fim de primavera e verão mas com uma maior variabilidade entre inverno e primavera. Entretanto uma completa caracterização destes ambientes implica em uma análise ao longo de um ano.
2. A análise multivariada sugeriu uma relação estreita entre salinidade e a taxa de respiração do mexilhão. Este fato ressalta a importância em investir esforços nos testes laboratoriais e confirmação de suas tendências.

3. A relação inversa observada entre as taxas de crescimento e as taxas de filtração e assimilação chamam a atenção para um parâmetro adicional a ser investigado (produção de folículos embrionários) e ainda para ajustes metodológicos do estudo (uso de organismos menores para aclimatação em campo e redução deste tempo de aclimatação para 15 dias ou menos).

4. A metodologia aplicada ressaltou a importância dos dados ambientais obtidos em fina escala temporal, destacando a importância do Projeto de Monitoramento Voluntário Olho Vivo (CTTMar-UNIVALI) e em especial a ampla aplicação dos dados de material em suspensão.

\section{REFERÊNCIAS}

Bayne, B.C. \& R.C. Newell. 1983. Physiological energetics of marine molluscs. In: Saleuddin, A.S.M. \& K.M. Wilbur (eds.) The mollusca. Vol. 4 Physiology, part. 1. Academic Press, New York. $407-515 p$.

Beers, J.R. 1976. Determination of zooplankton biomass. In: Steelman, W.F. 
(ed.) Zooplankton fixation and presenvation. Paris UNESCO, 35-84 $\mathrm{p}$.

Duarte, A.K. \& C. Resgalla Jr. 1996. Novo registro para duas espécies de crustáceos marinhos (Copepoda: Monstrilloida) na região sul do Brasil. XXI Congr. Bras. Zool. (Resumos).

EPA. 1989. Biomonitoring for control of toxicity in effluent discharges to the marine environment. EPA 625 8-89 015. 57pp.

Fernandes, F.C. 1981. Aspectos biológicos e ecológicos do mexilhão Perna perna (Linné, 1758) da região do Cabo Frio Brasil. Tese de Doutorado, Inst. de Biociências da USP. 91 pp.

Grant, J. \& P.J. Cranford. 1991. Carbon and nitrogen scope for growth as a function of diet in the sea Scallop Placopecten magellanicus. J. Mar. Biol. Ass. UK. 71:4155.

Kryger, J. \& H.V. Riisgard. 1988. Filtration rate capacities in 6 species of European freshwater bivalves. Oecologia. 77:34-38.

Navarro, J.M. 1988. The effects of salinity on the physiological ecology of Cloromytilus clorus (Molina, 1782) (Bivalvia: Mytilidae). J. Exp. Mar. Biol. Ecol. 122:19-33.

Newell, R.C. 1979. Biology of intertidal animals fators affecting the rate of feeding. In: Marine ecological surveys. Ltd. Wymberg South Africa. 459-502p.

Omori, M. \& T. Ikeda. 1984. Methods in marine zooplancton ecology. John Wiley \& Sons. New York. 332pp.

Pielou, E.C. 1984. The interpretation of ecological data. A primer on classification and ordination. Wiley Inter. Publ. John Wiley \& Sons, New York. 263pp.

Strickland, J.D.H. \& T.R. Parsons. 1972. A practical handbook of seawater analysis. Fish. Res. Board. Canada. 310 pp.

Villiers, C.J.; Allanson, B.R. \& A.N. Hodgson. 1989. The effect of temperature on the filtration rate of Solen cylindraceus (Hanley) (Mollusca: Bivalvia). S. Afr. J. Zool. 24(1):11-17.

Werner, I. \& J.T. Hollibaugh. 1993. Potamocorbula amurensis: comparison of clearance rates and assimilation efficiensis for phytoplankton and bacterioplankton. Limnol. Oceanogr. 38(5):949-964.

Widdows, J. \& D. Johnson, 1988. Physiological energetics of Mytilus edulis: Scope for Growth. Mar. Ecol. Prog. Ser. 46:113-121.

Wildish, D.J. \& M.P. Miyares. 1990. Filtration rate of blue mussels as a function of flow velocity: preliminary experiments. J. Exp. Mar. Biol. Ecol. 142:213-219.

Winter, J.E. 1973. The filtration rates of Mytilus edulis and its dependence on algal concentration, measured by a continuous automatic recording apparatus. Mar. Biol. 22:317-328.

Winter, J.E. 1978. A review on the knowledge of suspension-feeding in lamellibranchiate bivalves, with special reference to artificial aquaculture systems. Aquaculture. 13:1-33.

Wojciechowski Jr., E. 1997. Monitoramento do índice de condição do mexilhão Perna perna (Linné, 1758) em cultivo suspenço no parque de mitilicultura na enseada da Armação do Itapocoroy - Penha - SC Brasil. Monografia, Univ. do Vale do Itajaí. 